\title{
Utilização da madeira de Cryptomeria japonica para produção de painéis de partículas orientadas (OSB)
}

\author{
Use of Cryptomeria japonica wood for \\ oriented strand board production (OSB)
}

\author{
Rosilani Trianoski ${ }^{1}$, Setsuo Iwakiri ${ }^{1}$ e Daniel Chies ${ }^{2}$
}

\begin{abstract}
Resumo
Estudos relativos ao potencial tecnológico de novas espécies, bem como aos parâmetros do processo produtivo no setor de painéis de madeira são fundamentais, tendo em vista a importância deste segmento. Neste sentido, o objetivo deste trabalho foi avaliar a viabilidade de utilização da Cryptomeria japonica para a produção de painéis de partículas orientadas, assim como, estudar os efeitos da massa específica do painel, teor de resina e composição das camadas sobre a qualidade do produto final. Foram produzidos 8 tratamentos em um delineamento inteiramente casualizado, com arranjo fatorial $(2 \times 2 \times 2)$, cujos fatores foram: massa especifica nominal $\left(0,60\right.$ e $\left.0,70 \mathrm{~g} / \mathrm{cm}^{3}\right)$, teor de resina fenol formaldeído $(4 \%$ e $6 \%)$ e composição percentual das camadas (20-60-20 e 30-40-30). Os painéis foram prensados com temperatura de $180^{\circ} \mathrm{C}$, pressão de $4 \mathrm{MPa}$, e tempo de 8 minutos. Os resultados indicaram que os painéis produzidos com esta espécie, e com os parâmetros citados, possuem estabilidade dimensional e propriedades mecânicas satisfatórias, de acordo com os requisitos mínimos da norma européia EN 300, para painéis OSB com diferentes utilizações, não estruturais e estruturais, e em condições secas e úmidas. Concluiu-se que a madeira de Cryptomeria japonica apresenta viabilidade técnica para a produção de painéis OSB; o aumento da massa específica dos painéis contribuiu para a melhoria da estabilidade dimensional e das propriedades mecânicas; o teor de resina não exerceu grande influência sobre as propriedades; e a composição das camadas 20-60-20 gerou maior balanceamento das propriedades de flexão.
\end{abstract}

Palavras-chave: Painéis de madeira, EN 300, fenol-formaldeido.

\begin{abstract}
Studies on the technological potential of new species, and the parameters of the production process in the wood panel industry, are critical in view the importance of this segment. In this context, the objective this study was to evaluate the feasibility of using the Cryptomeria japonica for oriented strand board production, as well as, study the effects of panel density, resin content and composition of the layers on the quality of the final product. Eigth treatments were produced in a completely randomized design, with factorial arrangement $(2 \times 2 \times 2)$, whose factors were nominal density $\left(0.60\right.$ and $\left.0.70 \mathrm{~g} / \mathrm{cm}^{3}\right)$, phenol formaldehyde resin content ( $4 \%$ and $6 \%$ ), and percentage composition of the layers (20-60-20 and 30-40-30). The panels were pressed at $180^{\circ} \mathrm{C}$ of temperature, $4 \mathrm{MPa}$ of pressure and 8 minutes time. The results showed that panels produced with this specie and with the parameters mentioned have satisfactory dimensional stability and mechanical properties in accordance with the minimum requirements of European Standard EN 300 for OSB panels with different uses, non-structural and structural, and in dry and humid conditions. It was concluded that wood Cryptomeria japonica presented technical feasibility for OSB production; increasing the density of the panels contributed to improving the dimensional stability and mechanical properties; the resin content not exercised great influence on the properties; and the composition of the layers 20-60-20 generated greater balancing of bending properties.
\end{abstract}

Keywords: Wood panels, EN 300, phenol-formaldehyde.

\section{INTRODUÇÃO}

Nos últimos anos, a produção e o consumo de painéis de madeira reconstituída apresentaram o maior crescimento médio anual dentre os produtos florestais do Brasil. Segundo a ABIPA (2015), esse aumento se deve ao incremento do consumo doméstico e aos investimentos expressivos realizados pelas indústrias, as quais dobraram a capacidade nominal instalada na última década.

${ }^{1}$ Professor(a) Adjunto(a) do Departamento de Engenharia e Tecnologia Florestal. UFPR - Universidade Federal do Paraná. Av. Lothário Meissner 632 - Jardim Botânico - 80210170 - Curitiba, PR, Brasil. E-mail: rosillani@gmail.com.

2Engenheiro Florestal, Mestre em Engenharia Florestal. Madem Reels Group. Rod RSC 470 Km 223.82 - Caixa Postal 206 95720-000 - Garibaldi, RS, Brasil.95720-.000. Email: daniel@madem.com.br

Sci. For., Piracicaba, v. 44, n. 110, p. 487-496, jun. 2016 DOI: dx.doi.org/10.18671/scifor.v44n110.21 
Entre os diversos tipos de painéis de madeira, destaca-se o painel de partículas orientadas (Oriented Strand Board - OSB), disponível no mercado mundial desde o início da década de 80 (MENDES, 2010). No Brasil, mais precisamente em Ponta Grossa - PR, este tipo de painel começou a ser produzido comercialmente em 2002, pela Masisa, indústria com capacidade produtiva de $350.000 \mathrm{~m}^{3}$ / ano (VIDAL; HORA, 2014). Segundo estes autores, desde sua introdução no mercado brasileiro, o consumo de OSB segue em patamares menores, ao contrário do enorme crescimento registrado na América do Norte e Europa, onde estão localizadas a maioria das plantas industriais. Especialmente nos Estados Unidos e Canadá, a produção é em grande escala, e o consumo expressivo, principalmente pelo fato de que nestes países, $90 \%$ das construções empregam o sistema de construção a seco (steel framing e wood framing). Este tipo de painel, é ainda, utilizado na fabricação de móveis estofados, pallet e outros tipos de embalagens (LP, 2015).

De acordo com Maloney (1993), vários são os fatores que influenciam a qualidade final destes painéis, sendo os mais importantes, aqueles inerentes à madeira e ao processo produtivo, pois suas possíveis variações afetarão o comportamento das propriedades físicas e mecânicas.

Um dos fatores mais importantes inerentes à madeira é a espécie, onde espécies de baixa massa especifica são indicadas, pois possibilitam uma boa razão de compactação e a produção de painéis dentro da faixa adequada de massa especifica. Espécies com baixo teor de extrativos também são desejadas, pois altos valores destes componentes ocasionam problemas durante a prensagem, interferindo na cura do adesivo e gerando menor qualidade de colagem entre as partículas (MALONEY, 1993; CLOUTIER, 1998; MENDES, 2010). No Brasil, as espécies mais utilizadas para produção de OSB são as do gênero Pinus, especialmente o Pinus taeda (MENDES et al., 2002; IWAKIRI et al., 2004; MENDES, 2010). Entretanto, Iwakiri et al. (2004) ressaltam a importância da utilização de espécies alternativas de rápido crescimento, visando aumentar a oferta de matéria prima para uso das indústrias de base florestal.

Assim, uma espécie promissora para esta finalidade é a Cryptomeria japonica, pertencente à família Taxodiaceae. No país, foi introduzida há décadas, sendo plantada principalmente nos estados de São Paulo e no Sul de Minas Gerais (CARPANEZZI; CARVALHO, 1988). Destaca-se pelo seu rápido crescimento, grande potencial para plantios nas regiões altas e frias, boa adaptação ao clima e solo da região Sul, e boa qualidade da madeira (SANTOS et al., 2000). Possui massa especifica básica de $0,356 \mathrm{~g} / \mathrm{cm}^{3}$, a qual é adequada para painéis particulados (TRIANOSKI et al., 2013), além da utilização em serrados em geral (SANTOS et al., 2000), painéis compensados (PINTO, 2011) e para aplicações mais nobres como movelaria (PEREIRA et al., 2003).

Quanto aos fatores inerentes ao processo, pode-se citar a massa específica dos painéis, resina, composição de camadas, razão de compactação, entre outros. A massa específica influencia significativamente as propriedades físico-mecânicas (MALONEY, 1993), onde a faixa ideal para a produção está entre 0,65 a $0,70 \mathrm{~g} / \mathrm{cm}^{3}$ (ZHOW, 1990), e a mais utilizada, no Canadá, varia de 0,63 a $0,67 \mathrm{~g} / \mathrm{cm}^{3}$ (CLOUTIER, 1998). A massa específica do painel, juntamente com a massa específica da madeira, resultam na razão de compactação, onde, valores entre 1,3 a 1,6 são requeridos para que ocorra uma adequada densificação (KELLY, 1977; MALONEY, 1993, CLOUTIER, 1998; IWAKIRI, 2005).

Em relação à resina, a mais utilizada é a fenol-formaldeído, no entanto, a difenilmetano di-isocianato (MDI) também é empregada, principalmente na camada interna (CLOUTIER, 1998). O teor de resina aplicada varia de 3 a $6 \%$, onde maior teor, normalmente resulta em painéis de maior estabilidade dimensional, assim como, maior resistência mecânica (IWAKIRI, 2005). Já, a composição estrutural do painel em camadas cruzadas, melhora a resistência mecânica e a estabilidade dimensional nos sentidos paralelo e perpendicular (IWAKIRI, 2005), onde, segundo Cloutier (1998), a proporção ideal é na faixa de 20:60:20 a 30:40:30, baseado no peso de partículas.

Neste contexto, considerando as afirmações de Iwakiri et al. (2004), sobre a importância da utilização de espécies alternativas de rápido crescimento na indústria madeireira a fim de aumentar a oferta de matéria prima, bem como, as informações de Maloney (1993) e Cloutier (1998) a respeito dos fatores de influência sobre a qualidade dos painéis OSB, esta pesquisa teve como objetivo avaliar a viabilidade de utilização da Cryptomeria japonica para a produção de painéis OSB, assim como, estudar os efeitos da massa especifica do painel, teor de resina e composição das camadas sobre a qualidade do produto final. 


\section{MATERIAL E MÉTODOS}

A madeira de Cryptomeria japonica utilizada para a produção dos painéis OSB é proveniente de um plantio florestal com 35 anos de idade, de propriedade da empresa MADEM Reels Group, localizado em Rio Negrinho - SC. Foram selecionadas 10 árvores (DAP médio: 28,81 cm; altura total média: 21,14 m; e, altura comercial média: 17,97 m), as quais foram seccionadas de acordo com a altura comercial para obtenção de discos e toras $(2,50 \mathrm{~m})$. Os discos foram utilizados para a determinação da massa específica básica, a qual foi efetuada segundo os procedimentos recomendados pela norma COPANT (1972).

As toras foram desdobradas obtendo-se material representativo (blocos) de todas as posições do fuste para geração das partículas strands. As partículas foram geradas em picador de disco, com dimensões nominais de $85 \mathrm{~mm}$ de comprimento, $25 \mathrm{~mm}$ de largura e 0,7 $\mathrm{mm}$ de espessura. Após a geração, as partículas foram secas em estufa convencional com temperatura de $80{ }^{\circ} \mathrm{C}$, até teor de umidade de 3\% (base seca) e, posteriormente, classificadas em peneira com abertura de 9,52 $\mathrm{mm}$ (ASTM 3/8") para remoção de finos. Os painéis foram produzidos em triplicata, com dimensões de $480 \times 480 \times 15 \mathrm{~mm}, 4$ e $6 \%$ de resina fenol-formaldeído (teor de sólidos de 51,57\%, viscosidade de $503 \mathrm{cP}, \mathrm{pH}=12,66$ ) e 1\% de parafina (teor de sólidos de 28\%), conforme delineamento apresentado na Tabela 1. A parafina e a resina foram aplicadas separadamente pelo método de aspersão em encoladeira rotativa.

Tabela 1. Delineamento experimental.

Table 1. Experimental design.

\begin{tabular}{|c|c|c|c|}
\hline Tratamento & ME nominal $\left(\mathrm{g} / \mathrm{cm}^{3}\right)$ & Teor de resina (\%) & Composição das camadas (\%) \\
\hline 1 & 0,60 & 4 & $20-60-20$ \\
\hline 2 & 0,60 & 4 & $30-40-30$ \\
\hline 3 & 0,60 & 6 & $20-60-20$ \\
\hline 4 & 0,60 & 6 & $30-40-30$ \\
\hline 5 & 0,70 & 4 & $20-60-20$ \\
\hline 6 & 0,70 & 4 & $30-40-30$ \\
\hline 7 & 0,70 & 6 & $20-60-20$ \\
\hline 8 & 0,70 & 6 & $30-40-30$ \\
\hline
\end{tabular}

Os painéis foram prensados com temperatura de $180^{\circ} \mathrm{C}$, pressão específica de $4 \mathrm{MPa}$ e tempo de prensagem de 8 minutos. Após a prensagem, foram esquadrejados e acondicionados em câmara climatizada com temperatura de $20 \pm 2^{\circ} \mathrm{C}$ e umidade relativa de $65 \pm 5 \%$, até atingirem a umidade de equilíbrio. Na sequência, foram seccionados para obtenção dos corpos de prova dos ensaios físicos e mecânicos. Os ensaios físicos seguiram os procedimentos das normas EN (2002d) e EN (2002b), respectivamente para massa específica e absorção de água e inchamento em espessura após 2 e 24 de imersão em água, sendo utilizados 6 corpos de prova por painel. A razão de compactação foi calculada pela razão entre densidade do painel pela densidade da madeira. Os ensaios mecânicos efetuados foram flexão estática (EN, 2002a), a qual utilizou 6 corpos de prova no sentido paralelo e 3 no sentido perpendicular por painel, e tração perpendicular à superfície (EN, 2002c), com 6 amostras por painel.

O delineamento estatístico utilizado foi o inteiramente casualizado com arranjo fatorial $(2 \times 2 \times 2)$, cujos fatores foram: massa específica, teor de resina e composição das camadas. Os dados foram submetidos aos testes de Grubbs para avaliar a ocorrência de outliers, Shapiro Wilks para verificação da normalidade, Bartlett para homogeneidade de variância e Análise de variância. Quando rejeitada a hipótese de nulidade, foi aplicada a comparação de médias de Tukey, à 95\% de probabilidade. Os resultados médios foram comparados com os requisitos mínimos da norma EN (2006).

\section{RESULTADOS E DISCUSSÃO}

\section{Massa especifica da madeira}

A madeira de Cryptomeria japonica apresentou massa específica básica média de 0,356 g/ $\mathrm{cm}^{3}$, com variação de $12,23 \%$. Este resultado classifica a madeira como leve ou de baixa massa específica por 
apresentar valor de até $0,500 \mathrm{~g} / \mathrm{cm}^{3}$, segundo a classificação sugerida por Melo et al. (1990), e dentro da faixa de variação desejável para a produção de painéis OSB, conforme sugerido por Cloutier (1998), que é de 0,250 a 0,450 g/ $\mathrm{cm}^{3}$. Segundo Maloney (1993), madeiras de massa específica mais baixa possibilitam a produção de painéis dentro da faixa adequada de densidade e, resultam, normalmente, em painéis com resistência superior à painéis de espécies de massas específicas mais altas.

\section{Propriedades físicas dos painéis}

A Tabela 2 apresenta os resultados médios das propriedades físicas dos painéis.

Tabela 2. Resultados médios das propriedades físicas.

Table 2. Average results of physical properties.

\begin{tabular}{|c|c|c|c|c|c|c|}
\hline Tratamento & $\begin{array}{c}\text { ME } \\
\left(\mathrm{g} / \mathrm{cm}^{3}\right)\end{array}$ & RC & $\begin{array}{c}\text { AA 2h } \\
(\%)\end{array}$ & $\begin{array}{c}\text { AA 24h } \\
(\%)\end{array}$ & $\begin{array}{c}\text { IE 2h } \\
(\%)\end{array}$ & $\begin{array}{c}\text { IE 24h } \\
(\%)\end{array}$ \\
\hline $\begin{array}{l}1 \\
0,60 / 4 \% / 20-60-20\end{array}$ & $\begin{array}{l}0,576 \mathrm{~b} \\
(12,46)\end{array}$ & $\begin{array}{l}1,619 \mathrm{~cd} \\
(12,46)\end{array}$ & $\begin{array}{c}17,16 \mathrm{ab} \\
(32,82)\end{array}$ & $\begin{array}{c}52,07 \mathrm{ab} \\
(23,09)\end{array}$ & $\begin{array}{l}2,80 \mathrm{ab} \\
(38,64)\end{array}$ & $\begin{array}{c}12,63 a b \\
(34,61)\end{array}$ \\
\hline $\begin{array}{l}2 \\
0,60 / 4 \% / 30-40-30 \\
\end{array}$ & $\begin{array}{l}0,586 \mathrm{~b} \\
(13,89)\end{array}$ & $\begin{array}{c}1,646 \text { bcd } \\
(13,89)\end{array}$ & $\begin{array}{l}20,51 \text { a } \\
(41,26)\end{array}$ & $\begin{array}{l}55,83 \text { a } \\
(30,68)\end{array}$ & $\begin{array}{c}3,16 \text { a } \\
(37,72)\end{array}$ & $\begin{array}{l}13,76 \text { a } \\
(26,23)\end{array}$ \\
\hline $\begin{array}{l}3 \\
0,60 / 6 \% / 20-60-20\end{array}$ & $\begin{array}{l}0,595 \mathrm{~b} \\
(13,04)\end{array}$ & $\begin{array}{c}1,754 \mathrm{bc} \\
(13,04)\end{array}$ & $\begin{array}{c}19,24 \mathrm{ab} \\
(58,50)\end{array}$ & $\begin{array}{c}46,46 \mathrm{ab} \\
(37,40)\end{array}$ & $\begin{array}{l}2,60 \mathrm{ab} \\
(38,57)\end{array}$ & $\begin{array}{l}9,39 \mathrm{bc} \\
(24,64)\end{array}$ \\
\hline $\begin{array}{l}4 \\
0,60 / 6 \% / 30-40-30 \\
\end{array}$ & $\begin{array}{l}0,566 \mathrm{~b} \\
(10,38)\end{array}$ & $\begin{array}{l}1,589 \mathrm{~d} \\
(10,39)\end{array}$ & $\begin{array}{c}16,94 \mathrm{ab} \\
(15,22)\end{array}$ & $\begin{array}{c}48,15 \mathrm{ab} \\
(13,39)\end{array}$ & $\begin{array}{l}1,71 \mathrm{~b} \\
(36,83)\end{array}$ & $\begin{array}{l}7,92 \mathrm{c} \\
(31,15) \\
\end{array}$ \\
\hline $\begin{array}{l}5 \\
0,70 / 4 \% / 20-60-20\end{array}$ & $\begin{array}{l}0,633 \mathrm{a} \\
(11,30)\end{array}$ & $\begin{array}{c}1,778 \mathrm{ab} \\
(11,29)\end{array}$ & $\begin{array}{c}15,79 \mathrm{ab} \\
(35,00)\end{array}$ & $\begin{array}{c}44,54 \mathrm{ab} \\
(24,06)\end{array}$ & $\begin{array}{c}1,86 \mathrm{~b} \\
(32,90)\end{array}$ & $\begin{array}{l}9,83 \mathrm{bc} \\
(26,00)\end{array}$ \\
\hline $\begin{array}{l}6 \\
0,70 / 4 \% / 30-40-30 \\
\end{array}$ & $\begin{array}{l}0,644 \mathrm{a} \\
(10,68)\end{array}$ & $\begin{array}{l}1,810 \mathrm{ab} \\
(10,68)\end{array}$ & $\begin{array}{l}11,99 \mathrm{~b} \\
(48,13)\end{array}$ & $\begin{array}{l}40,02 \mathrm{~b} \\
(27,49)\end{array}$ & $\begin{array}{l}2,79 a b \\
(29,38)\end{array}$ & $\begin{array}{c}11,01 \mathrm{abc} \\
(26,03)\end{array}$ \\
\hline $\begin{array}{l}7 \\
0,70 / 6 \% / 20-60-20 \\
\end{array}$ & $\begin{array}{c}0,658 \text { a } \\
(9,94) \\
\end{array}$ & $\begin{array}{c}1,904 a \\
(9,92) \\
\end{array}$ & $\begin{array}{c}13,56 \mathrm{ab} \\
(39,26) \\
\end{array}$ & $\begin{array}{c}42,25 \mathrm{ab} \\
(23,77)\end{array}$ & $\begin{array}{l}2,51 \mathrm{ab} \\
(47,08) \\
\end{array}$ & $\begin{array}{l}9,31 \mathrm{bc} \\
(31,17)\end{array}$ \\
\hline $\begin{array}{l}8 \\
0,70 / 6 \% / 30-40-30\end{array}$ & $\begin{array}{l}0,638 a \\
(11,97)\end{array}$ & $\begin{array}{l}1,792 a b \\
(11,97)\end{array}$ & $\begin{array}{c}16,46 \mathrm{ab} \\
(27,09)\end{array}$ & $\begin{array}{c}42,69 \mathrm{ab} \\
(20,58)\end{array}$ & $\begin{array}{l}2,50 \mathrm{ab} \\
(38,98)\end{array}$ & $\begin{array}{c}8,25 \mathrm{c} \\
(24,84)\end{array}$ \\
\hline
\end{tabular}

ME: massa específica; RC: razão de compactação; AA: Absorção de água; IE: Inchamento em espessura; Médias seguidas de mesma letra na mesma coluna são estatisticamente iguais pelo teste de Tukey a $95 \%$ de probabilidade; Valores entre parênteses referem-se ao coeficiente de variação das amostras.

A massa especifica média dos tratamentos variou de 0,566 a $0,595 \mathrm{~g} / \mathrm{cm}^{3}$ para os painéis produzidos com massa específica nominal de $0,60 \mathrm{~g} / \mathrm{cm}^{3}$ e, de 0,633 a $0,658 \mathrm{~g} / \mathrm{cm}^{3}$ para os painéis produzidos com massa específica de $0,70 \mathrm{~g} / \mathrm{cm}^{3}$, evidenciando que as perdas foram parecidas entre os diferentes tratamentos e, quando comparadas com a massa específica nominal, os tratamentos produzidos com $0,70 \mathrm{~g} / \mathrm{cm}^{3}$ foram os que mais se distanciaram do valor desejado. Este resultado pode ser explicado pela maior quantidade de partículas utilizadas em painéis de maior massa especifica, pelo maior retorno em espessura após liberação da pressão, assim como, pela liberação das tensões de prensagem durante o processo de climatização. Constatou-se também, diferença estatística significativa de massa específica entre os tratamentos com massa específica nominal distinta, o que era esperado pois, a diferenciação foi determinada no cálculo de massa.

Os valores médios da razão de compactação apresentaram-se elevados, em função, principalmente, da massa específica da madeira de Cryptomeria japonica e, na maioria dos casos, superiores a faixa descrita como ideal por Maloney (1993), de 1,3 a 1,6, o que implica em contato satisfatório entre as partículas, influenciando positivamente e, principalmente, nas propriedades de flexão.

Em relação à absorção de água, o tratamento 6, produzido com massa específica nominal de $0,70 \mathrm{~g} / \mathrm{cm}^{3}, 4 \%$ de resina e composição das camadas de 30-40-30, apresentou o menor valor médio, no entanto, só difere estatisticamente do tratamento 2, que foi produzido com o mesmo teor de resina, mesmo percentual de camadas e massa específica nominal de $0,60 \mathrm{~g} / \mathrm{cm}^{3}$. Desta forma, para esta propriedade, pode-se verificar que a massa específica exerceu maior influência sobre a absorção, principalmente em gerar um painel com menos espaços vazios na sua estrutura, dificultando a entrada e acúmulo de água.

Para o inchamento em espessura, observa-se que o tratamento 4, produzido com massa específica nominal de $0,60 \mathrm{~g} / \mathrm{cm}^{3}, 6 \%$ de resina e proporção de camadas de 30-40-30, apresentou o melhor desempenho em termos absolutos, diferindo apenas do tratamento 2 no tempo de imersão de 2 horas e, dos tratamentos 1 e 2, quando ao tempo de imersão de 24 horas. Para esta propriedade, e 
de acordo com os tratamentos citados, nota-se o maior efeito do teor de resina, onde uma maior quantidade de resina é responsável pelo maior recobrimento da partícula e melhor qualidade de colagem, inibindo o inchamento.

Foram observados elevados valores do coeficiente de variação para as propriedades de estabilidade dimensional, os quais podem ser classificados como muito alto (acima de 30\%), segundo Gomes (1985). Esta elevada variabilidade nas propriedades de absorção de água e inchamento em espessura muitas vezes são comuns em painéis particulados experimentais devido à dificuldades do controle do processo produtivo. Os valores deste trabalho são compatíveis com Mendes (2001) que obteve coeficientes de até 45,97\%, aos de Saldanha e Iwakiri (2009) que encontraram valores de até $32,17 \%$, e aos de Iwakiri et al. (2014), cujo coeficiente de variação chegou a 43,81\%.

Considerando o valor máximo da norma EN (2006) para inchamento em espessura após 24 horas de imersão, de painéis com espessura entre $10 \mathrm{~mm}$ a $18 \mathrm{~mm}$, observa-se que os tratamentos 3 a 8 atendem os requisitos para os tipos de painéis OSB 1 (painéis não estruturais de uso geral e interior); OSB 2 (painéis estruturais para uso em condições secas), OSB 3 (painéis estruturais para uso em condições úmidas) e OSB 4 (painéis estruturais de carregamento pesado para uso em condições úmidas), que exigem no máximo inchamento de 25\%, 20\%, 15\% e 12\%, respectivamente. Já os tratamentos 1 e 2 atendem os requisitos de painéis OSB 1, OSB 2 e OSB 3.

Em relação à painéis produzidos com Pinus, gênero mais utilizado para esta finalidade, verifica-se que os painéis OSB de Cryptomeria japonica foram superiores em termos de estabilidade dimensional, quando comparados com os resultados apresentados por Saldanha e Iwakiri (2009), que obtiveram valores de absorção de água de 30,38\% a 74,17\% e inchamento em espessura de 27,95\% a 42,78\% em painéis de Pinus taeda, massa especifica nominal de 0,65 a 0,90 g/ $\mathrm{cm}^{3}$ e proporção das camadas de 25-50-25\%; e aos de Mendes et al. (2003), que obtiveram valores entre 25\% a 66\% para absorção de água e de $20 \%$ a $42 \%$ para inchamento em espessura a partir de painéis de Pinus taeda com massas específicas nominais de 0,65 e $0,80 \mathrm{~g} / \mathrm{cm}^{3}$, teores de resina de 4 e $6 \%$ e diferentes composições de camadas, indicando desta forma, que esta espécie, embora possua madeira de menor massa específica, gera painéis mais estáveis.

Na Tabela 3 podem ser visualizados os resultados médios dos efeitos principais sobre as propriedades físicas.

Tabela 3. Resultados médios dos efeitos principais sobre as propriedades físicas.

Table 3. Average results of main effects on the physical properties.

\begin{tabular}{|c|c|c|c|c|c|c|}
\hline Tratamento & $\begin{array}{c}\mathrm{ME} \\
\left(\mathrm{g} / \mathrm{cm}^{3}\right) \\
\end{array}$ & RC & $\begin{array}{c}\text { AA 2h } \\
(\%)\end{array}$ & $\begin{array}{c}\text { AA 24h } \\
(\%)\end{array}$ & $\begin{array}{c}\text { IE 2h } \\
(\%) \\
\end{array}$ & $\begin{array}{c}\text { IE 24h } \\
(\%)\end{array}$ \\
\hline \multicolumn{7}{|c|}{ Fator: Massa específica } \\
\hline $0,60 \mathrm{~g} / \mathrm{cm}^{3}$ & $\begin{array}{l}0,588 \mathrm{~b} \\
(12,96)\end{array}$ & $\begin{array}{l}1,652 \mathrm{~b} \\
(12,96) \\
\end{array}$ & $\begin{array}{l}18,53 \text { a } \\
(41,37) \\
\end{array}$ & $\begin{array}{l}50,73 \text { a } \\
(28,50) \\
\end{array}$ & $\begin{array}{c}2,54 \mathrm{a} \\
(43,51) \\
\end{array}$ & $\begin{array}{l}10,84 a \\
(36,44) \\
\end{array}$ \\
\hline $0,70 \mathrm{~g} / \mathrm{cm}^{3}$ & $\begin{array}{l}0,648 \text { a } \\
(11,16)\end{array}$ & $\begin{array}{l}1,820 \mathrm{a} \\
(11,15)\end{array}$ & $\begin{array}{l}14,47 \mathrm{~b} \\
(40,24)\end{array}$ & $\begin{array}{l}42,34 \mathrm{~b} \\
(23,77)\end{array}$ & $\begin{array}{c}2,38 \mathrm{a} \\
(39,99)\end{array}$ & $\begin{array}{c}9,60 \mathrm{~b} \\
(28,53)\end{array}$ \\
\hline \multicolumn{7}{|c|}{ Fator: Teor de resina } \\
\hline $4 \%$ & $\begin{array}{l}0,610 \text { a } \\
(12,83)\end{array}$ & $\begin{array}{l}1,713 \text { a } \\
(12,83)\end{array}$ & $\begin{array}{l}16,49 \text { a } \\
(44,86)\end{array}$ & $\begin{array}{c}46,66 \text { a } \\
(28,10)\end{array}$ & $\begin{array}{c}2,41 \text { a } \\
(41,78)\end{array}$ & $\begin{array}{l}10,24 \text { a } \\
(31,58)\end{array}$ \\
\hline $6 \%$ & $\begin{array}{l}0,626 \text { a } \\
(12,98) \\
\end{array}$ & $\begin{array}{l}1,757 \text { a } \\
(12,98) \\
\end{array}$ & $\begin{array}{l}16,39 \text { a } \\
(41,03) \\
\end{array}$ & $\begin{array}{l}46,47 \text { a } \\
(28,04) \\
\end{array}$ & $\begin{array}{r}2,52 \mathrm{a} \\
(41,53) \\
\end{array}$ & $\begin{array}{l}10,21 a \\
(35,26) \\
\end{array}$ \\
\hline \multicolumn{7}{|c|}{ Fator: Composição das camadas } \\
\hline $20-60-20$ & $\begin{array}{l}0,627 \text { a } \\
(12,89)\end{array}$ & $\begin{array}{l}1,760 \text { a } \\
(12,89)\end{array}$ & $\begin{array}{l}16,29 \text { a } \\
(45,05)\end{array}$ & $\begin{array}{l}46,03 \text { a } \\
(28,33)\end{array}$ & $\begin{array}{c}2,40 a \\
(42,24) \\
\end{array}$ & $\begin{array}{l}10,15 a \\
(31,82)\end{array}$ \\
\hline $30-40-30$ & $\begin{array}{l}0,610 \text { a } \\
(12,87) \\
\end{array}$ & $\begin{array}{l}1,710 \mathrm{a} \\
(12,88) \\
\end{array}$ & $\begin{array}{l}16,39 \text { a } \\
(41,03)\end{array}$ & $\begin{array}{l}46,66 \text { a } \\
(28,03) \\
\end{array}$ & $\begin{array}{c}2,52 \mathrm{a} \\
(41,53) \\
\end{array}$ & $\begin{array}{l}10,21 \mathrm{a} \\
(35,26)\end{array}$ \\
\hline
\end{tabular}

ME: massa específica; RC: razão de compactação; AA: Absorção de água; IE: Inchamento em espessura; Médias seguidas de mesma letra na mesma coluna, para cada fator avaliado, são estatisticamente iguais pelo teste de Tukey a $95 \%$ de probabilidade; Valores entre parênteses referem-se ao coeficiente de variação das amostras.

Verifica-se que o fator massa específica afetou significativamente a razão de compactação e, que ambos, influenciaram as propriedades de absorção de água e inchamento em espessura. O aumento da massa específica contribuiu de forma positiva para a redução destas e, consequentemente, para o aumento da estabilidade dimensional. Normalmente, painéis com maior massa especifica possuem estrutura mais fechada, impedindo a entrada e acúmulo da água. Por outro lado, maior massa 
específica e maior razão de compactação implicam em maior liberação das tensões de prensagem, favorecendo o inchamento, fato que não foi observado neste estudo, e que pode estar, de alguma forma, associado a composição química da madeira, mais precisamente aos extrativos, que nesta espécie apresenta valor médio de 5,9\% (PEREIRA et al., 2003) e que pela presença de algum componente específico pode ter inibido o inchamento.

Em relação ao fator teor de resina, nota-se que houve um ligeiro aumento da massa específica do painel, no entanto, não foi constatada diferença estatística significativa. Observa-se ainda, que esta variável não exerceu influência significativa nas demais propriedades físicas, concluindo-se, portanto, que é possível maximizar a produção em termos de quantidade de adesivo, caso o fator de decisão seja a estabilidade dimensional.

Para o fator composição das camadas, também não foi evidenciada influência estatisticamente significativa sobre as propriedades físicas, indicando que a produção de painéis OBS, a partir desta espécie, pode ser realizada em ambas as proporções, e que esta variável não interfere na estabilidade dimensional.

\section{Propriedades mecânicas dos painéis}

A Tabela 4 apresenta os resultados médios das propriedades mecânicas dos diferentes tratamentos propostos no delineamento experimental.

Tabela 4. Resultados médios das propriedades mecânicas.

Table 4. Average results of mechanical properties.

\begin{tabular}{|c|c|c|c|c|c|}
\hline \multirow{3}{*}{ Tratamento } & \multicolumn{2}{|c|}{ Paralelo } & \multicolumn{2}{|c|}{ Perpendicular } & \multirow{2}{*}{ TP } \\
\hline & MOR & MOE & MOR & MOE & \\
\hline & (MPa) & (MPa) & (MPa) & (MPa) & (MPa) \\
\hline $\begin{array}{l}1 \\
0,60 / 4 \% / 20-60-20\end{array}$ & $\begin{array}{l}19,77 \mathrm{c} \\
(31,94)\end{array}$ & $\begin{array}{c}3.845 \mathrm{bc} \\
(23,61)\end{array}$ & $\begin{array}{c}20,04 \mathrm{ab} \\
(29,96)\end{array}$ & $\begin{array}{l}2.385 a \\
(15,99)\end{array}$ & $\begin{array}{l}0,52 \mathrm{bc} \\
(14,25)\end{array}$ \\
\hline $\begin{array}{l}2 \\
0,60 / 4 \% / 30-40-30\end{array}$ & $\begin{array}{l}19,63 \mathrm{c} \\
(64,96) \\
\end{array}$ & $\begin{array}{l}3.020 \mathrm{c} \\
(57,75) \\
\end{array}$ & $\begin{array}{l}12,29 \mathrm{~b} \\
(52,52) \\
\end{array}$ & $\begin{array}{l}1.627 \mathrm{a} \\
(67,93)\end{array}$ & $\begin{array}{l}0,45 \mathrm{c} \\
(22,14) \\
\end{array}$ \\
\hline $\begin{array}{l}3 \\
0,60 / 6 \% / 20-60-20 \\
\end{array}$ & $\begin{array}{c}27,04 \mathrm{bc} \\
(28,85)\end{array}$ & $\begin{array}{c}4.385 \mathrm{bc} \\
(20,10)\end{array}$ & $\begin{array}{c}24,80 \mathrm{ab} \\
(8,13)\end{array}$ & $\begin{array}{l}2.453 \mathrm{a} \\
(15,70)\end{array}$ & $\begin{array}{l}0,59 \mathrm{ab} \\
(14,17)\end{array}$ \\
\hline $\begin{array}{l}4 \\
0,60 / 6 \% / 30-40-30 \\
\end{array}$ & $\begin{array}{c}29,52 \mathrm{bc} \\
(30,51)\end{array}$ & $\begin{array}{c}4.458 \mathrm{bc} \\
(19,99)\end{array}$ & $\begin{array}{l}13,14 \mathrm{~b} \\
(39,81) \\
\end{array}$ & $\begin{array}{l}1.509 a \\
(36,73)\end{array}$ & $\begin{array}{l}0,62 \mathrm{ab} \\
(11,37)\end{array}$ \\
\hline $\begin{array}{l}5 \\
0,70 / 4 \% / 20-60-20\end{array}$ & $\begin{array}{c}31,46 \text { abc } \\
(23,52)\end{array}$ & $\begin{array}{c}4.808 \mathrm{ab} \\
(12,67)\end{array}$ & $\begin{array}{c}21,30 \mathrm{ab} \\
(20,52)\end{array}$ & $\begin{array}{l}2.069 \mathrm{a} \\
(15,33)\end{array}$ & $\begin{array}{l}0,52 \mathrm{bc} \\
(16,93)\end{array}$ \\
\hline $\begin{array}{l}6 \\
0,70 / 4 \% / 30-40-30\end{array}$ & $\begin{array}{l}35,33 \mathrm{a} \\
(25,28)\end{array}$ & $\begin{array}{l}5.207 \mathrm{a} \\
(21,58)\end{array}$ & $\begin{array}{c}17,78 \mathrm{ab} \\
(20,93)\end{array}$ & $\begin{array}{l}1.746 \mathrm{a} \\
(21,44)\end{array}$ & $\begin{array}{l}0,49 \text { bc } \\
(24,40)\end{array}$ \\
\hline $\begin{array}{l}7 \\
0,70 / 6 \% / 20-60-20\end{array}$ & $\begin{array}{c}33,58 \mathrm{ab} \\
(17,19)\end{array}$ & $\begin{array}{c}4.714 \mathrm{ab} \\
(17,55)\end{array}$ & $\begin{array}{l}24,92 \mathrm{a} \\
(23,18)\end{array}$ & $\begin{array}{l}2.588 a \\
(26,56)\end{array}$ & $\begin{array}{l}0,59 a b \\
(20,20)\end{array}$ \\
\hline $\begin{array}{l}8 \\
0,70 / 6 \% / 30-40-30\end{array}$ & $\begin{array}{l}35,94 \mathrm{a} \\
(25,87)\end{array}$ & $\begin{array}{l}5.391 \mathrm{a} \\
(20,33)\end{array}$ & $\begin{array}{c}16,24 \mathrm{ab} \\
(9,56)\end{array}$ & $\begin{array}{l}1.857 \mathrm{a} \\
(10,01)\end{array}$ & $\begin{array}{l}0,69 \mathrm{a} \\
(6,05)\end{array}$ \\
\hline
\end{tabular}

MOR: Módulo de ruptura; MOE: Módulo de elasticidade; TP: Tração perpendicular; Médias seguidas de mesma letra na mesma coluna são estatisticamente iguais pelo teste de Tukey a 95\% de probabilidade; Valores entre parênteses referem-se ao coeficiente de variação das amostras.

Os resultados indicaram que o tratamento de maior resistência e rigidez média à flexão no sentido paralelo foi o tratamento 8 , produzido com massa específica nominal de $0,70 \mathrm{~g} / \mathrm{cm}^{3}$, $6 \%$ de resina e proporção de camadas 30-40-30, e que é estatisticamente igual aos tratamentos produzidos com mesma massa especifica nominal. Já no sentido perpendicular, o tratamento 7 se destacou, sendo este, estatisticamente diferente apenas dos tratamentos 2 e 4 no módulo de ruptura. Para o módulo de elasticidade não foi observada diferença estatística significativa.

Considerando a norma EN (2006), verifica-se que os tratamentos 1 e 2 atendem os requisitos mínimos de $18 \mathrm{MPa}$ e $2.500 \mathrm{MPa}$ e, $9 \mathrm{MPa}$ e $1.200 \mathrm{MPa}$, para MOR e MOE nos sentidos paralelo e perpendicular, respectivamente, sendo classificados como do tipo OSB 1, podendo ser utilizados como painéis não estruturais de uso geral e interior seco. Já os tratamentos 3, 4, 6 e 8, além de atender os requisitos mínimos do OSB tipo 1, atingem os valores mínimos do OSB tipo 2 e OSB tipo 3 (MOR paralelo: $20 \mathrm{MPa}$, MOE paralelo: $3.500 \mathrm{MPa}$, MOR perpendicular: $10 \mathrm{MPa}$ e MOE perpendicular: $1.400 \mathrm{MPa}$ ), podendo ser utilizados estruturalmente em condições secas e úmidas. E os tratamentos 5 e 7, atingem, além dos tipos anteriormente citados, os requisitos mínimos do 
OSB tipo 4, cujas resistência e rigidez mínimas são de $28 \mathrm{MPa}$ e 4.800 MPa para o sentido paralelo e, de $15 \mathrm{MPa}$ e $1.900 \mathrm{MPa}$, para o sentido perpendicular, respectivamente, caracterizando-se como painéis estruturais de carregamento pesado para uso em condições úmidas.

Em relação à tração perpendicular, o tratamento 8 apresentou o maior valor médio $(0,69 \mathrm{MPa})$, sendo este, estatisticamente igual ao tratamentos 3, 4 e 7, que são os tratamentos com a maior quantidade de resina (6\%). Considerando o requisito mínimo da norma européia, verifica-se que todos os tratamentos atingiram o valor mínimo do OSB tipo 4, de 0,45 $\mathrm{MPa}$, indicando que podem ser utilizados, quanto a esta propriedade, em finalidades estruturais e não estruturais, submetidas tanto à condições secas quanto úmidas.

Comparando com painéis de Pinus, os resultados obtidos no presente trabalho foram ligeiramente inferiores aos de Mendes et al. (2003) que obtiveram valores de 40,31 a 50,21 MPa e de 23,63 a 36,48 MPa para MOR nos sentidos paralelo e perpendicular, respectivamente, e de 4.469 a 51.918 MPa e 1.995 a $2.152 \mathrm{MPa}$ para MOE nos respectivos sentidos; e também aos de Saldanha e Iwakiri (2009), cujos valores variaram na faixa de 28,58 a 53,94 MPa e 19,95 a 30,97 MPa para MOR paralelo e perpendicular, e 5,128 a $7.041 \mathrm{MPa}$ e 2.721 a $3028 \mathrm{MPa}$ para MOE paralelo e perpendicular, respectivamente. Quanto à tração perpendicular, os valores obtidos por estes autores foram de 0,57 a 0,81 MPa (MENDES et al., 2003), e de 0,22 a 0,50 MPa (SALDANHA; IWAKIRI, 2009), os quais são similares aos encontrados nesta pesquisa.

Tabela 5. Resultados médios dos efeitos principais sobre as propriedades mecânicas.

Table 5. Average results of main effects on the mechanical properties.

\begin{tabular}{|c|c|c|c|c|c|}
\hline \multirow{3}{*}{ Tratamento } & \multicolumn{2}{|c|}{ Paralelo } & \multicolumn{2}{|c|}{ Perpendicular } & \multirow{2}{*}{ TP } \\
\hline & MOR & MOE & MOR & MOE & \\
\hline & (MPa) & (MPa) & (MPa) & (MPa) & (MPa) \\
\hline \multicolumn{6}{|c|}{ Fator: Massa específica } \\
\hline $0,60 \mathrm{~g} / \mathrm{cm}^{3}$ & $\begin{array}{l}24,01 \mathrm{~b} \\
(41,28)\end{array}$ & $\begin{array}{l}3.914 \mathrm{~b} \\
(32,63)\end{array}$ & $\begin{array}{l}17,09 a \\
(40,01)\end{array}$ & $\begin{array}{l}1.936 \text { a } \\
(37,81)\end{array}$ & $\begin{array}{c}0,54 \mathrm{a} \\
(19,51)\end{array}$ \\
\hline $0,70 \mathrm{~g} / \mathrm{cm}^{3}$ & $\begin{array}{l}34,73 \text { a } \\
(25,13) \\
\end{array}$ & $\begin{array}{l}5.099 \text { a } \\
(19,57) \\
\end{array}$ & $\begin{array}{l}20,21 \mathrm{a} \\
(25,32) \\
\end{array}$ & $\begin{array}{l}2.087 \mathrm{a} \\
(24,53) \\
\end{array}$ & $\begin{array}{r}0,56 \text { a } \\
(21,92) \\
\end{array}$ \\
\hline \multicolumn{6}{|c|}{ Fator: Teor de resina } \\
\hline $4 \%$ & $\begin{array}{l}27,72 \text { a } \\
(45,66)\end{array}$ & $\begin{array}{l}4.238 a \\
(35,87) \\
\end{array}$ & $\begin{array}{l}18,37 a \\
(31,63)\end{array}$ & $\begin{array}{l}1.995 a \\
(30,07)\end{array}$ & $\begin{array}{c}0,49 \mathrm{~b} \\
(20,14)\end{array}$ \\
\hline $6 \%$ & $\begin{array}{l}30,95 \text { a } \\
(27,59) \\
\end{array}$ & $\begin{array}{r}4.743 a \\
(20,69) \\
\end{array}$ & $\begin{array}{l}19,05 \text { a } \\
(34,71) \\
\end{array}$ & $\begin{array}{r}2.037 \text { a } \\
(32,10) \\
\end{array}$ & $\begin{array}{r}0,61 \mathrm{a} \\
(14,87) \\
\end{array}$ \\
\hline \multicolumn{6}{|c|}{ Fator: Composição das camadas } \\
\hline $20-60-20$ & $\begin{array}{l}26,76 \mathrm{~b} \\
(29,72)\end{array}$ & $\begin{array}{l}4.387 a \\
(20,19)\end{array}$ & $\begin{array}{l}22,31 \mathrm{a} \\
(23,20)\end{array}$ & $\begin{array}{l}2.362 a \\
(19,90)\end{array}$ & $\begin{array}{c}0,56 \mathrm{a} \\
(17,31)\end{array}$ \\
\hline $30-40-30$ & $\begin{array}{l}31,69 \text { a } \\
(38,63)\end{array}$ & $\begin{array}{l}4.590 a \\
(33,56)\end{array}$ & $\begin{array}{c}14,84 \mathrm{~b} \\
(30,44)\end{array}$ & $\begin{array}{l}1.671 \mathrm{~b} \\
(33,33)\end{array}$ & $\begin{array}{c}0,54 \mathrm{a} \\
(23,97)\end{array}$ \\
\hline
\end{tabular}

MOR: Módulo de ruptura; MOE: Módulo de elasticidade; TP: Tração perpendicular; Médias seguidas de mesma letra na mesma coluna, para cada fator avaliado, são estatisticamente iguais pelo teste de Tukey a $95 \%$ de probabilidade; Valores entre parênteses referem-se ao coeficiente de variação das amostras.

De acordo com a Tabela 5, o fator massa especifica afetou positivamente todas as propriedades mecânicas, no entanto, foram constatadas diferenças estatísticas significativas apenas para o MOR e o MOE no sentido paralelo. Este resultado é corroborado pela afirmação de Maloney (1993) e Kelly (1977), que relatam que painéis com maior massa específica apresentam maior resistência mecânica, e atribuem esta vantagem à maior quantidade de partículas de madeira e, consequentemente, à maior densificação e compactação durante o processo de prensagem.

Em relação ao fator teor de resina, verifica-se que o aumento desta variável de $4 \%$ para $6 \%$, proporcionou melhoria em todas as propriedades, onde o aumento mais acentuado foi observado na tração perpendicular, sendo constatada diferença estatística significativa. Este fato, se deve a maior disponibilidade de resina por área superficial de partículas, melhorando com isso, as ligações inter-partículas (IWAKIRI, 2005), entretanto, Kelly (1977) relata que acima de um determinado nível, o aumento do teor de resina não atua mais na melhoria da ligação entre as partículas, somente eleva o recobrimento da partícula. 
Quanto ao fator composição das camadas, a proporção face-miolo-face 30-40-30 favoreceu as propriedades de flexão estática no sentido paralelo e, a proporção 20-60-20, o sentido perpendicular. Este comportamento é justificado, principalmente, pela disposição das partículas em relação ao plano do painel e o respectivo plano de ensaio. Mendes et al. (2003) complementam que este fato ocorre porque o aumento da espessura do miolo favorece o comportamento do MOE perpendicular, em função das partículas do miolo estarem também nesta direção. Ainda, o resultado obtido para este fator, indica que a proporção 20-60-20 apresentou melhor balanceamento das propriedades de flexão estática nos diferentes sentidos, sendo condizente com o estudo de Iwakiri et al. (2003).

\section{CONCLUSÕES}

A madeira de Cryptomeria japonica apresenta viabilidade técnica para a produção de painéis de partículas orientadas - OSB.

O aumento da massa específica dos painéis contribuiu para a melhoria da estabilidade dimensional, assim como, das propriedades mecânicas.

O aumento do teor de resina, de $4 \%$ para $6 \%$, não exerceu influência sobre as propriedades de estabilidade dimensional.

O aumento do teor de resina, de 4\% para 6\%, proporcionou um ligeiro aumento nos módulos de ruptura e elasticidade, e um aumento mais acentuado na tração perpendicular.

As diferentes composições das camadas não afetou a estabilidade dimensional.

A composição das camadas 20-60-20 favoreceu as propriedades de flexão no sentido perpendicular, a composição 30-40-30 favoreceu a flexão no sentido paralelo e, as diferentes composições não afetaram a tração perpendicular. A composição 20-60-20 gerou melhor balanceamento das propriedades de flexão.

Os tratamentos 1 e 2 podem ser classificados como painéis OSB tipo 1 (uso não estrutural geral seco), os tratamentos 3, 4, 6 e 8 como painéis OSB tipo 1, 2 e 3 (uso não estrutural seco e uso estrutural seco e úmido), e os tratamentos 5 e 7 , além dos anteriormente citados, atingem o requisito mínimo da classificação OSB tipo 4 (uso estrutural pesa em condições úmidas).

\section{AGRADECIMENTOS}

Os autores expressam seus agradecimentos à Madem Reels Group pela doação da madeira, e à Momentive pela doação da resina fenol-formaldeído.

\section{REFERÊNCIAS BIBLIOGRÁFICAS}

ABIPA. ASSOCIAÇÃO BRASILEIRA DE INDÚSTRIAS DE PAINÉIS DE MADEIRA. Disponível em: < http:// www.abipa.org.br >. Acesso em: 18 abr. 2015.

CARPANEZZI, A.; CARVALHO, P. E. R. Zoneamento ecológico para plantios florestais do Estado de Santa Catarina. Curitiba: Embrapa CNPF, 1988. 103 p. (Documentos, 21)

CLOUTIER, A. Oriented strandboard (OSB): raw material, manufacturing process, properties of wood-base fiber and particle materials. In: INTERNATIONAL SEMINAR ON SOLID WOOD PRODUCTS OF HIGH TECHNOLOGY. 1998, Belo Horizonte. Anais... Viçosa: SIF, 1998, p. 173-185.

COPANT. COMISSÃO PANAMERICANA DE NORMAS TÉCNICAS. COPANT 461: Maderas - Determinación del peso especifico aparente. 1972.

EN. EUROPEAN STANDARD. EN 300: Oriented Strand Boards (OSB) - Definitions, classification and specifications. Bruxelas: 2006.

EN. EUROPEAN STANDARD. EN 310: Determination of modulus of elasticity in bending and of bending strength. Bruxelas; 2002a. 
EN. EUROPEAN STANDARD. EN 317: Determination of swelling in thickness after immersion in water. Bruxelas; 2002b.

EN. EUROPEAN STANDARD. EN 319: Determinação da resistência à tração perpendicular às faces da placa. Bruxelas; 2002c.

EN. EUROPEAN STANDARD. EN 323: Determinação da massa volúmica. Bruxelas; 2002d.

GOMES, F. P. Curso de estatística experimental. 11.ed. São Paulo: Nobel, 1985. 467 p.

IWAKIRI, S. Painéis de madeira reconstituída. Curitiba: FUPEF. 2005. 247 p.

IWAKIRI, S.; MENDES, L. M.; SALDANHA, L. K. Produção de chapas de partículas orientadas "OSB" de Eucalyptus grandis com diferentes teores de resina, parafina e composição em camadas. Ciência Florestal, Santa Maria, v. 13, n. 1, p. 89-94, 2003.

IWAKIRI, S.; MENDES, L. M.; SALDANHA, L. K.; SANTOS, J.C. Utilização da madeira de eucalipto na produção de chapas de partículas orientadas - OSB. Cerne, Lavras, v. 10, n.1, p. 46-52, 2004.

IWAKIRI, S.; POTULSKI, D. C.; SANCHES, F. G.; SILVA, J. B.; TRIANOSKI, R.; PRETKO, W. C. Avaliação do potencial de uso da madeira de Acrocarpus fraxinifolius, Grevilea robusta, Melia azedarach e Toona ciliata para produção de painéis OSB. Cerne, Lavras, v. 20, n. 2, p. 277-284, 2014.

KELLY, M. W. A critical literature review of relationships between processing parameters and physical properties of particleboards. Madison: USDA Forest Service, 1977. 66 p. (FLP General Technical Report, 10)

LP BUILDING PRODUCTS. LP Brasil: Indústria de Painéis OSB. Disponível em: < http://www. http://www. lpbrasil.com.br >. Acesso em: 04 nov. 2015.

MALONEY, T. M. Modern particleboard e dry-process fiberboard manufacturing. 2.ed São Francisco: Miller Freeman Publication, 1993. 689 p.

MELO, J. E.; CORADIN, V. T. R.; MENDES, J. C. Classes de densidade para madeiras da Amazônia Brasileira. In: CONGRESSO FLORESTAL BRASILEIRO, 6. 1990, Campos do Jordão, Anais... São Paulo: SBS/SBEF, 1990. v. 3, p. 695-699.

MENDES, L. M. Pinus spp. na produção de painéis de partículas orientadas (OSB). 2001. 103 p. Tese (Doutorado em Ciências Florestais) - Universidade Federal do Paraná, Curitiba, 2001.

MENDES, L. M.; IWAKIRI, S.; MATOS, J. L. M.; KEINERT, S.; SALDANHA, L. K. Efeitos da densidade, composição dos painéis e teor de resina nas propriedades de painéis OSB. Floresta e Ambiente, Seropédica, v. 10, n. 1, p. 01-17, 2003.

MENDES, L. M.; IWAKIRI, S.; MATOS, J.L.M.; KEINERT, S.; SALDANHA, L.K. Pinus spp. na produção de painéis de partículas orientadas (OSB). Ciência Florestal, Santa Maria, v. 12, n. 2, p. 135-145, 2002.

MENDES, R. F. Efeito do tratamento térmico sobre as propriedades de painéis OSB. 2010. 115p. Dissertação (Mestrado em Ciências) - Escola Superior de Agricultura "Luiz de Queiroz", Universidade de São Paulo, Piracicaba, 2010.

PEREIRA, J. C. D.; HIGA, R. C. V.; SHIMIZU, J. Y. Propriedades da madeira do cedrinho japonês. Colombo: Embrapa Florestas, 2003. 4 p. (Comunicado Técnico, 88). 
PINTO, J. A. Análise da viabilidade do uso da madeira de "Cryptomeria japonica" para laminação e produção de painéis compensados. 2011. 92 p. Dissertação (Mestrado em Engenharia Florestal) - Universidade Federal do Paraná, Curitiba, 2011.

SALDANHA, L. K.; IWAKIRI, S. Influência da densidade e do tipo de resina nas propriedades tecnológicas de painéis osb de Pinus taeda L. Floresta, Curitiba, v. 39, n. 3, p. 571-576, 2009.

SANTOS, C. B.; LONGHI, S. J.; HOPPE, J. M.; MOSCOVICH, S. A. Efeito do volume de tubetes e tipos de substratos na qualidade de mudas de Cryptomeria japonica (L.F.) D. Don. Ciência Florestal, Santa Maria, v. 10, n. 2 , p. $1-15,2000$.

TRIANOSKI, R.; IWAKIRI, S.; MATOS, J. L. M.; CHIES, D. Utilização da madeira de Cryptomeria japonica para produção de painéis aglomerados. Scientia Forestalis, Piracicaba, v. 41, n. 97, p. 057-064, 2013.

VIDAL, A. C. F.; HORA, A. B. Panorama de mercado: painéis de madeira. BNDES Setorial, Rio de Janeiro, $\mathrm{n}$. 40, p. 323-384, 2014.

ZHOW, D. A study of oriented structural board made from hybrid poplar. Physical and mechanical properties of OSB. Holz Als Roh Und Werkstoff, Berlin, v. 48, n. 7-8, p. 293-296, 1990.

Recebido em 20/07/2015

Aceito para publicação em 16/12/2015 